testable question as to whether this is its role in indirect flight muscle. In the wild, flies rarely have the opportunity for an 'athletic' pre-flight warm-up, because they require instantaneous maximum power. It seems likely either that significant MLC phosphorylation must occur within milliseconds or that the phosphorylation state will have to be maintained at optimal levels during adult life - begging the question as to whether phosphorylation of the MLC has a regulatory or a modulatory role in insect indirect flight muscles.

John C. Sparrow is in the Department of Biology, University of York, York Y01 5DD, UK.

\section{The heart of the matter}

\section{GerryK. Skinner}

\section{ACCORDING to Robert Frost ${ }^{1}$ \\ We dance round in a ring and suppose, But the Secret sits in the middle and knows}

but as we dance, we do get some hints about just what it is that sits in the centre of our Galaxy. Whatever is there is totally hidden from optical telescopes; interstellar dust clouds that lie in the plane of the Milky Way are thought to attenuate visible light by a factor of $10^{11}$. Radio, infrared and X-ray telescopes can peer through the clouds. The trouble is that the stories they tell seem conflicting. Narayan et $\mathrm{al}^{2}$ may now have found a way of reconciling the existence of a massive black hole in the galactic nucleus, as implied by some observations, with the relative paucity of the high-energy emission that might be expected from such an object. On page 623 of this issue, they show how in some circumstances such a black hole can devour a surprisingly large fraction of the gravitational energy released by matter attracted to it.

Ironically, in many ways we know more about the inner regions of other galaxies than we do of our own galactic nucleus. A unified model $^{3}$ is evolving which links a wide variety of active galactic nuclei (AGNs). At one extreme are highly luminous objects seen only in distant parts of the Universe and with appropriately exotic names (quasars, blazars and optically violent variables, to name but a few). At the other are 'Seyfert' galaxies, which are dominated by a bright point-like nucleus, and even galaxies that are normal except for an unusual source of energy at their core. All are seen as members of one extended family.

Many of the differences between the various types of AGN are explained as merely the effect of seeing similar objects from particular directions. Thus blazars are recognized as quasars in which one of the jets of relativistic particles is directed almost exactly towards us, boosting the apparent luminosity and giving the illusion of superluminal motion of the blobs of ejected particles. Similarly, two types of Seyferts are now widely thought to differ only in whether the orientation of a dust-filled molecular cloud forming a torus around the nucleus is such as to allow us a direct view in or whether we see only scattered radiation.

Some AGNs have jets and associated radio lobes whereas others do not, but most of the remaining differences seem to be in the luminosity of the central 'engine' thought to be at the core of all these objects, which ranges from less than $10^{35}$ watts to more than $10^{40}$ watts. This central engine is generally thought to involve a massive black hole (MBH). The evidence is strongest in the case of quasars, where one can deduce that the emitting region must have a size less than 0.1 light years and a mass greater than $10^{9}$ solar masses $\left(M_{\odot}\right)$. Part of the potential energy released when matter approaches the event horizon of an $\mathrm{MBH}$ escapes as radiation and provides the power. Most calculations indicate that a fraction $q \approx 0.1$ of the rest mass energy $m c^{2}$ of accreted matter escapes in this way. An accretion rate of a few tens of $M_{\odot}$ per year, which can credibly be supplied by surrounding gas and by stars torn apart in the gravitational field, will provide $>10^{40}$ watts and explain even the highest quasar luminosities.

Occam's razor and the economics of the automobile industry both suggest that where possible one should use the same components in the 'top of the range' models and in their less powerful brethren. So most models of Seyferts, and even of galaxies showing the slightest sign of activity in their nuclei, also tend to be based on accreting massive black holes.

It seems that in the past a higher fraction of galaxies were active and it has been suggested that many, perhaps most, galaxies go through phases where they show at least some nuclear activity. Once a galaxy develops an $\mathrm{MBH}$ at its core, it can never regain its virginity. So what of the core of our own, comparatively placid, Galaxy? Is there a central $\mathrm{MBH}$ engine, perhaps in a run-down or dormant state or in miniature form?

The motion of the stars and gas clouds immediately surrounding the nucleus of our Galaxy is most easily explained by supposing that they are moving in the gravitational field around a central mass of about $10^{6} M_{\odot}$ within a radius of 0.5 light years ${ }^{4}$, although no individual observations actually require such a mass. Remarkably, radio observations of the region reveal a compact, non-thermal, radio source termed $\mathrm{Sgr} \mathrm{A}^{*}$, which is unique in our Galaxy. It lies exactly within an arcsecond - where an $\mathrm{MBH}$ would be expected if one exists. Sgr A* is only about the size of the orbit of Jupiter 5 and its (projected) velocity is less than 27 $\mathrm{km} \mathrm{s}^{-1}$, substantially lower than would be typical of an object of stellar mass moving in the gravitational fields of the region, so indicating a high mass (see ref. 6).

Thus there is circumstantial evidence that our galactic nucleus houses an $\mathrm{MBH}$. The surprise is that $\mathrm{Sgr} \mathrm{A}^{*}$ is not a conspicuous object outside the radio waveband. Looking at infrared maps, the main centre of activity is the star group IRS16, an arcsecond away. There are still only hints of faint infrared emission ${ }^{7}$ from Sgr A*. And although there is variable $\mathrm{X}$-ray emission from the region, presumably originating in $\mathrm{Sgr} \mathrm{A}^{*}$, it is far weaker than expected ${ }^{8}$.

What Narayan et al. have now done is to produce a model for Sgr $\mathrm{A}^{*}$ in which a black hole of mass $7 \times 10^{5} M_{\odot}$ accretes matter but in which most of the energy is advected onto the $\mathrm{MBH}$. The trick is that if the accretion rate is not too high the local cooling time can exceed the inflow time - the matter gets very hot but is swallowed before it has time to radiate much energy. With only a couple of free parameters to twiddle they predict a spectrum which matches that of Sgr A* remarkably well, from the radio band through to $\mathrm{X}$ - and $\gamma$-rays.

Of course, just because a model is consistent with observations it doesn't have to be the right one. With the Hubble Space Telescope and ground-based observations both finding evidence for large concentrated central masses in more and more galaxies, including comparatively nearby ones, there may be ample opportunity for testing the idea in a range of circumstances.

Gerry K. Skinner is in the Space Reseach Group, School of Physics and Space Research, University of Birmingham, Edgbaston, Birmingham B15 2TT, UK.

1. Frost, R. The Secret Sits (from Complete Works, Cape, London, 1951)

2. Narayan, R., Yi, I. \& Mahadevan, R. Nature 374, 623-625 (1995).

3. Antonucci, R. A. Rev. Astr. Astrophys. 31, 473-521 (1993).

Genzel, R. Hollenbach, D. \& Townes, C. H. Rep. Prog. Phys. 57, 417--479 (1994).

Krichbaum, T. P etal. Astr. Astrophys. 274, L37-L40 (1993).

Blitz, L., Binney, J., Lo, K. Y., Bally, J. \& Ho, P. T. P. Nature 361, 417-424 (1993).

7. Liu, T., Becklin, E. E., Henry, J. P. \& Simons, D. Astr. J. 106, 1484-1489 (1993).

8. Predehl, P. \& Trümper, J. Astr. Astrophys. 290, L29-L32 (1994). 